\title{
Robo1/Robo4: Differential expression of angiogenic markers in colorectal cancer
}

\author{
JÖRN GRÖNE ${ }^{1}$, OLIVER DOEBLER ${ }^{1}$, CHRISTOPH LODDENKEMPER ${ }^{2}$, \\ BIRGIT HOTZ ${ }^{1}$, HEINZ-JOHANNES BUHR ${ }^{1}$ and SARAH BHARGAVA ${ }^{1}$ \\ ${ }^{1}$ Department of Surgery, ${ }^{2}$ Institute of Pathology, Charité - Medical School Berlin, \\ Campus Benjamin Franklin, Hindenburgdamm 30, D-12200 Berlin, Germany
}

Received December 6, 2005; Accepted February 9, 2006

\begin{abstract}
The family of roundabout (Robo) proteins is related to the transmembrane receptors and plays a major role in the process of axonal guidance in neurogenesis. It has recently been shown that Robo proteins are also associated with tumor angiogenesis with Slit2 acting as the corresponding ligand. The aim of this study was to validate the differential expression by means of microarray analysis and real-time PCR and to analyze the in situ expression of Robo1 and Robo4 in colorectal cancer. Quantitative analyses of Robo1, Robo4 and Slit2 mRNA expression measured by large scale gene expression studies (Affymetrix U133A) showed a significant up-regulation of Robo1 in tumor vs. normal tissue, whereas Robo4 and Slit2 showed no significant deregulation. For subsequent real-time PCR experiments, paired colorectal tissue samples from cancerous and corresponding noncancerous tissues were obtained from 50 colorectal cancer patients who underwent surgical resection. Robo1 mRNA overexpression in cancerous tissue compared with normal counterparts was observed in $80 \%$ of the patients with a 4-fold expression in $45 \%$ and a 12 -fold expression in $15 \%$. For Robo4, an up-regulation was detected in $>70 \%$ (36/50). For Slit2, no differential expression was observed. The overexpression of Robo1 and Robo4 in tumor vs. normal tissue was verified using real-time PCR. The histological analysis revealed an expression of Robo1 mainly in tumor cells, whereas Robo4 is located primarily in endothelial cells of tumor vessels. Therefore, the Robo proteins provide potential target structures for the anti-tumorigenic and anti-angiogenic therapy of colorectal carcinoma.
\end{abstract}

Correspondence to: Dr Jörn Gröne, Department of Surgery, Charité - Medical School Berlin, Campus Benjamin Franklin, Hindenburgdamm 30, D-12200 Berlin, Germany

E-mail: joern.groene@charite.de

Key words: colorectal cancer, roundabout protein, Robo1, Robo4, Slit2, angiogenesis, gene expression

\section{Introduction}

The Robos (roundabouts) comprise a family of single-pass transmembrane receptors identified in Drosophila, first isolated in 1998. Four different Robos (Robo1, Robo2, Robo3, Robo4) are described to date, whereas Robo1, 2 and 3 share structural homology containing five immunoglobulin (Ig) domains and three fibronectins in the extracellular region. In contrast, Robo4 is much smaller and consists of only three Igs and two fibronectin. Robo4 is also termed magic roundabout. So far, human slits are considered the corresponding candidate ligands for the Robo receptors. Slits are large secreted proteins and consist of a family of three genes, Slit1, Slit2 and Slit3. The Slit/Robo-interaction plays a critical role in neurogenesis by mediating a repulsive signal during the process of axonal guidance. This signalling pathway has also been shown to be important in myogenesis, inhibition of leukocyte migration and the development of tumors (1-3). Robo/Slit has been detected in several tumor cell lines and in primary tumors such as prostate carcinoma or breast cancer (3-5). It is indicated that Robo/Slit is involved in angiogenic mechanisms whereas endothelial cells expressing Robol receptors are attracted by Slit2 which is secreted by human tumor cells (3). Moreover, Wang and his co-workers successfully inhibited tumor growth by applying blocking antibodies into a xenograft animal model. The expression of Robo4 is limited to the endothelium and has been demonstrated to be strongly up-regulated in vessels of tumors in the brain, colon and bladder (6). Further investigation revealed an essential role for Robo4 in in vivo angiogenesis (7). Thus, the Robo receptors seem to be a promising target for anti-tumorigenic and anti-angiogenic therapy, respectively. For the assumed Robo receptor, Slit2, a suppressive role in colorectal cancer was assigned (8).

Colorectal cancer (CRC) remains a significant cause of cancer-related mortality in the Western world. As an alternative treatment modality to conventional therapies, there has been an increasing interest in recent years in developing angiosuppressive agents. The inhibition of angiogenesis is based on starving tumor cells of the blood supply which is required for tumor growth and represents a reasonable approach in the therapeutic treatment of cancer. Several anti-angiogenic agents alone, or in combination with conventional therapy modalities, are now in preclinical trials, such as protease inhibitors, 
suppressors of angiogenic factors or direct inhibitors of endothelial cell proliferation (9).

However, it has not been determined whether Robo1, Robo4 or Slit 2 may also play a critical role in colorectal cancer or even in tumor angiogenesis. Therefore, our study sought to examine the expression level of these target genes in colorectal cancer tissue and normal background tissue at the mRNA level. Furthermore, we aimed to investigate the distribution of Robo1 and Robo4 in the cellular compartments of tissues.

\section{Materials and methods}

Patients and tissue preparation. Fifty patients with colorectal cancer undergoing elective curative surgery from 2001 to 2003 at the Department of Surgery, Charité, Campus Benjamin Franklin, Berlin, were prospectively recruited for this study with informed consent approved by the local ethics committee. Thirty out of 50 patients were UV-laser microdissected for chiparray-based expression analysis. Tumor and normal tissue from all 50 patients was macrodissected for RNA validation by means of real-time PCR.

Microdissection. Laser microdissection of snap frozen normal mucosa and corresponding primary colorectal carcinoma tissue specimens of 30 patients was performed as previously described (10). For molecular analysis, $\leq 100,000$ cells were pooled. Tumor and normal cells were obtained from different blocks from each case. Normal colorectal tissue was preferably taken from areas at the resection margin.

Macrodissection. Matched paired tissue samples from an additional $20 \mathrm{CRC}$ patients (a total of 50 patients) were cut (cryo sections of 6-8 $\mu \mathrm{m}$ ) and stained with hematoxylin and eosin (H\&E). Tumor and normal tissue were verified by an experienced clinician and relevant areas were marked. Slides $(20 \mu \mathrm{m})$ were prepared and macrodissected with a sterile scalpel according to the mark. A new template H\&E slide of 6-8 $\mu \mathrm{m}$ was prepared every $200 \mu \mathrm{m}$ to control tumor and normal cell areas.

RNA preparation and array hybridization. Poly $\mathrm{A}^{+}$RNA preparation and hybridization of the Affymetrix human genome U133 set (HG-U133) was performed as previously described (10).

Quantitative real-time PCR. RNA was isolated using NucleoSpin ${ }^{\circledR}$ RNA II (Macherey-Nagel, Düren, Germany) according to the manufacturer's instructions. Single-stranded cDNA was synthesized from $2 \mu \mathrm{g}$ total RNA with oligo dT primer and M-MLV reverse transcriptase (Promega, Mannheim, Germany). Real-time PCR was performed using the hybridization probe technique by means of a LightCycler system (Roche Diagnostics, Mannheim, Germany). All primers and hybridization probes were synthesized by TIB MolBiol (Berlin, Germany). As a housekeeping gene, B2-microglobulin was used (11). Reactions were performed in duplicates of $20 \mu 1$ reaction mixtures with $2 \mu 1$ template cDNA, $2.5 \mathrm{U}$ HotStarTaq Polymerase (Qiagen, Hilden, Germany), HotStarTaq PCR buffer, $2 \mathrm{mM} \mathrm{MgCl}_{2}$ and $10 \mathrm{mM}$ dNTPs. PCR reactions consisted of one initial enzyme activation step at $95^{\circ} \mathrm{C}$ for $15 \mathrm{~min}$ followed by 45 cycles at $95^{\circ} \mathrm{C}$ for $10 \mathrm{sec}$, annealing at $58^{\circ} \mathrm{C}$ for $30 \mathrm{sec}$ and amplification at $72^{\circ} \mathrm{C}$ for $30 \mathrm{sec}$ in the step acquisition mode. Crossing points for each reaction were defined by Second Derivative Maximum and arithmetic baseline adjustment.

Immunohistochemistry for Robol. Formalin-fixed, paraffinembedded sections $(3 \mu \mathrm{m})$ were treated with $0.01 \%$ EDTA $\mathrm{pH} 8$ in a microwave oven for $5 \mathrm{~min}$ at $800 \mathrm{~W}$ followed by $10 \mathrm{~min}$ at $200 \mathrm{~W}$. Endogenous alkaline phosphatase activity in sections was quenched by treatment with $1 \%$ hydrogen peroxide solution for $25 \mathrm{~min}$. After blocking slides for $5 \mathrm{~min}$ (Powerblock, BioGenex), anti-Robo1 antibody (Santa Cruz Biotechnology, Santa Cruz, CA) was applied in a 1:100 dilution and was incubated at $37^{\circ} \mathrm{C}$ for $30 \mathrm{~min}$. After thorough rinsing in PBS-Tween solution, slides were incubated with a 1:200 dilution of a biotinylated secondary anti-goat antibody (Santa Cruz Biotechnology) for 20 min followed by a 20-min incubation with streptavidin peroxidase. For color development, slides were incubated for $5 \mathrm{~min}$ with the chromogen DAB (3.3'-diaminobenzidine tetrahydrochloride). All samples were lightly counterstained with hematoxylin for $30 \mathrm{sec}$ before dehydration and mounting. Immunostaining was evaluated by two different observers [a clinician (O.D.) and a pathologist (C.L.)]. The estimated percentage of stained cells $(\%)$ and the average staining intensity of the positive cells (normal and tumor) were expressed as follows: (0) none; (+) weak; (++) intermediate; (+++) strong.

In situ hybridization for Robo4. The Robo4 cDNA representing the sequence from nucleotides 1252 to 1820 was amplified by PCR using specific oligonucleotide primers: sense 5'-AG CCAACTGGACTGTAGTTG-3' and antisense 5'-GAGCC ATAAAAAGTGCTGGTG-3' as described previously (12). Human Robo4 cDNA was used as a template. The amplified product was then subcloned into the pGEM cloning vector (Promega, Mannheim, Germany). The recombinant clones were isolated and sequenced to confirm the authenticity of the sequence and to determine orientation. Plasmid DNA was linearized with PstI restriction endonuclease to transcribe sense cRNA using T7 RNA polymerase and to transcribe antisense cRNA using SP6 RNA polymerase. The cRNA transcripts were digoxigenin-labeled by in vitro transcription using a DIG RNA labeling kit (Roach Diagnostics Corp., Indianapolis, IN) according to the manufacturer's recommendations. Probes were then assessed for quality by agarose gel electrophoresis followed by blotting onto a nylon membrane and visualization with alkaline phosphatase-linked anti-DIG antibodies and in situ hybridization color development reagents.

The protocol was adapted from the Roche Molecular Biochemicals publication Nonradioactive In situ Hybridization Application Manual. Paraffin-embedded tissues were cut into $1-\mu \mathrm{m}$ sections, placed on silane-coated slides and dried at $37^{\circ} \mathrm{C}$ overnight. To remove, paraffin sections were incubated in xylene overnight and rehydrated through a series of graded ethanol solutions. Slides were washed twice in TBS and permeabilized with $1 \mu \mathrm{g} / \mathrm{ml}$ RNase-free Proteinase $\mathrm{K}$ for $30 \mathrm{~min}$ at $37^{\circ} \mathrm{C}$ in TBS containing $2 \mathrm{mM} \mathrm{CaCl}_{2}$. Then slides were washed twice with TBS, incubated with $0.1 \mathrm{M}$ triethanolamine buffer, $\mathrm{pH} 8.0$, containing $0.25 \%(\mathrm{v} / \mathrm{v})$ acetic anhydride for $10 \mathrm{~min}$, and pre-hybridized at $44^{\circ} \mathrm{C}$ for $1 \mathrm{~h}$ with a pre-hybridization mix (Roche Applied Science) containing 


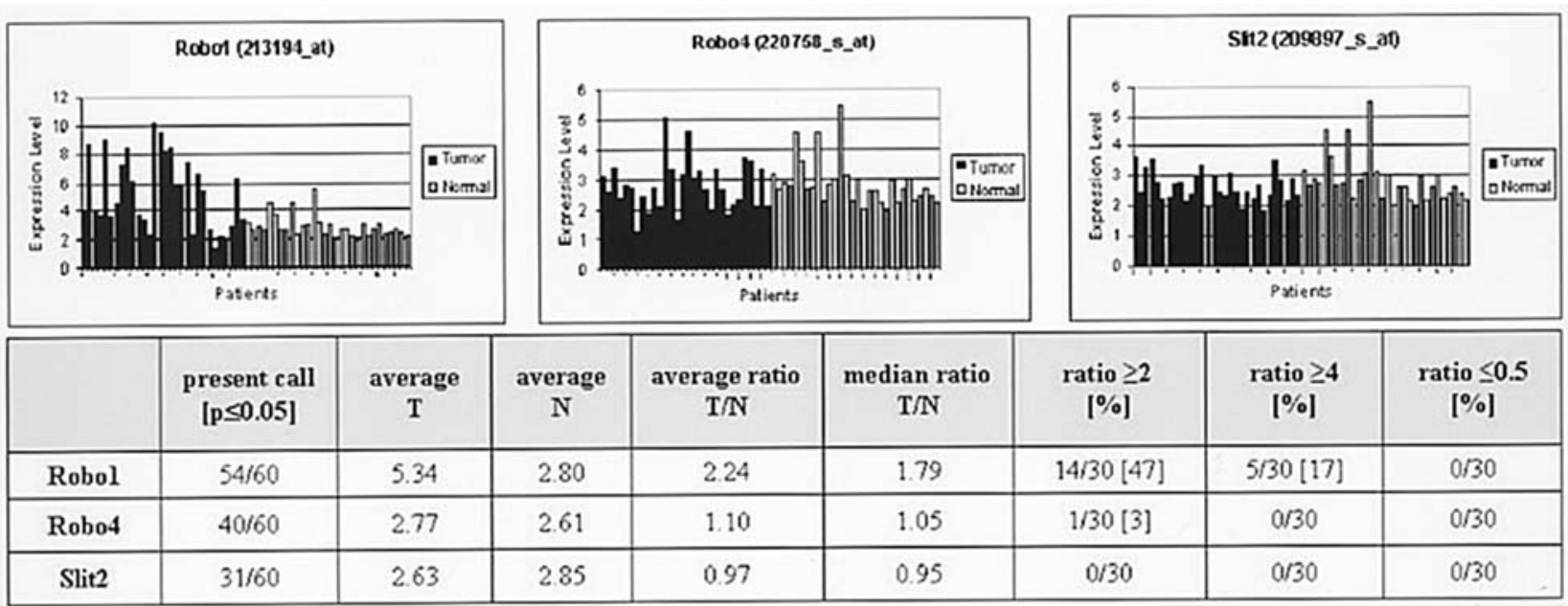

Figure 1. Relative expression data of Robo1, Robo4 and Slit2 of 30 matched colorectal tumor and corresponding normal tissues based on Affymetrix HG-U133 set expression analysis as linear schematic view and tabulated. For each case, normal (grey bar) and tumor (black bar), relative expression levels are shown. A probeset was called present and regarded as valid information if the difference between perfect match and mismatch was significant ( $\mathrm{p} \leq 0.05$; data not shown). Mean relative expression values of normal and tumor samples, the mean and median tumor-normal-foldchange and the ratio of FC $>2$, FC $>4$ and $\mathrm{FC}<0.5$ are listed.

$50 \%(\mathrm{v} / \mathrm{v})$ deionized formamide. Sections were hybridized overnight at $45^{\circ} \mathrm{C}$ in a hybridization buffer (Roche Applied Science) containing $5 \mu \mathrm{g} / \mathrm{ml}$ digoxigenin-labeled RNA. On the following day, slides were washed for $30 \mathrm{~min}$ in $2 \mathrm{X} \mathrm{SSC}$, $1 \mathrm{~h}$ in $1 \mathrm{X} \mathrm{SSC}, 1.5 \mathrm{~h}$ in $0.5 \mathrm{X} \mathrm{SSC}$ and $1 \mathrm{~h}$ in $1 \mathrm{X} \mathrm{SSC}$ at $53^{\circ} \mathrm{C}$. After that, sections were washed twice in $0.5 \mathrm{X}$ SSC for $10 \mathrm{~min}$ and in $0.2 \mathrm{X} \mathrm{SSC}$ for $10 \mathrm{~min}$. To visualize bound probe, sections were washed in TBS for $10 \mathrm{~min}$, and blocked with blocking buffer (10\% fetal calf serum in TBS) for $30 \mathrm{~min}$ at room temperature. The slides were incubated overnight at $4^{\circ} \mathrm{C}$ in 1:5000 dilution of anti-DIG Fab fragment (Roche Applied Science) in blocking buffer. Then sections were incubated with ready-to-use tablets of NBT/BCIP (nitro blue tetrazolium/5-bromo-4-chloro-3-indolyl phosphate) dissolved in $10 \mathrm{ml}$ aqua dest. for $5 \mathrm{~min}-48 \mathrm{~h}$ in the dark. The color reaction was terminated by washing slides with TBS. Coverslips were added to the developed slides with Aquapolymount (Polysciences). Serial sections were hybridized with identical quantities of sense cRNA as a control for specificity.

Statistical methods. Normalization of chiparray data and identification of differentially expressed genes were performed as previously described elsewhere (10). Relative quantification of PCR products was determined using Relative Quantification Software version 1.0 (Roche Diagnostics) (13). The relative expression was calculated using calibrator-normalized relative quantification including efficiency correction. Statistical analysis of LightCycler data was performed using SigmaPlot ${ }^{\circledR}$ 8.0 for Windows, including boxplot and t-test.

\section{Results}

Affymetrix U133A microarray-data. The probeset 213194_at for roundabout protein1 was called present $(\mathrm{p} \leq 0.05)$ in $54 / 60$ analyzed sample pairs. Robo1 was up-regulated no less than two-fold in $>47 \%$ of analyzed tumors with a mean tumor/ normal foldchange (FC) of 2.24. CRC $(>17 \%, 5 / 30)$ had a $>4$-fold overexpression compared to corresponding normal tissue. In none of the patients was Robo1 down-regulated with an FC of $<0.5$. The probeset for Robo4 (220758_s_at) was absent in two-thirds of the analyzed samples (20/60). An up-regulation in tumor tissue was only detected in one patient (FC 2.06) with a mean tumor/normal FC of 1.10 for all samples. There was no down-regulation of Robo4 in tumor tissue with an FC of $<0.5$. The probeset for Slit2 (209897_s_at) showed no significant deregulation in tumor vs. normal tissue (Fig. 1). There was no significant correlation between sex, tumor localization (colon vs. rectum), $\mathrm{T}$ stage and grading and gene expression (data not shown).

Validation study by means of quantitative real-time PCR (LightCycler). At the outset, a total of 50 colorectal cancer cases of 50 patients (Table I) were recruited for our study. The microarray expression data of Robol and Robo4 were verified using quantitative real-time PCR. Our results confirmed the up-regulation of these genes in samples of colorectal tumor tissue. Overall, Robo1 was overexpressed in $78 \%$ of the patients $(39 / 50)$ in the tumor endothelium compared to normal epithelium. In $42 \%$ of the patients $(21 / 50)$, a 4 -fold overexpression of Robo1 was demonstrated and, in $14 \%$ of the tumors (7/50), a 12-fold overexpression was demonstrated. For Robo4, up-regulation was found in $72 \%(36 / 50)$, whereas $44 \%$ of the patients $(22 / 50)$ revealed a 2 -fold and $16 \%(8 / 50)$ revealed a 4-fold overexpression in the tumor tissue. Quantification of Slit2 tends to be down-regulated in colorectal cancer tissue. In 68\% (34/50) of the cases, reduced Slit2 expression was detected in tumor tissue compared to normal tissue with $42 \%(21 / 50)$ displaying a half-fold underexpression. The patients $(32 \%)$ revealed moderate overexpression with only $16 \%(8 / 50)$ showing a 2-fold expression of Slit2 (Fig. 2).

Immunohistochemistry/in situ hybridization. Immunohistochemical analysis was performed by means of immunohistochemistry for Robo1 (commercially available antibody) and in situ hybridization for Robo1 and Robo4. IHC staining of paraffin-embedded tumor tissue of $10 \mathrm{CRC}$ patients out of 
Table I. Clinical and histopathological characteristics of 50 patients with oncologically resected sporadic colorectal cancer and no preceding neoadjuvant radiochemotherapy.

\section{UICC I UICC II UICC III UICC IV}

Patients (n)

11

15

14

10

50

Age at time of diagnosis

$63(38-87)$

$66(39-80)$

$60(22-84)$

$61(50-76)$

$63(22-87)$

(median, min, max in years)

Sex

$\begin{array}{llllll}\text { Female } & 5 & 7 & 6 & 5 & 23 \\ \text { Male } & 6 & 8 & 8 & 5 & 27\end{array}$

Localization of tumor

Colon

2

10

6

5

23

Rectum

9

5

8

5

$\mathrm{T}$ categories

T2

11

0

2

0

13

T3

0

15

T4

0

0

11

33

4

Grading

\begin{tabular}{llllll} 
Moderate & 9 & 6 & 6 & 3 & 24 \\
Low & 2 & 9 & 8 & 7 & 26 \\
\hline
\end{tabular}

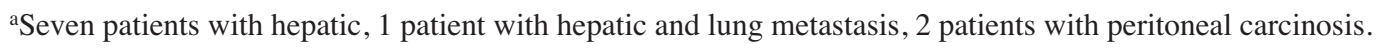
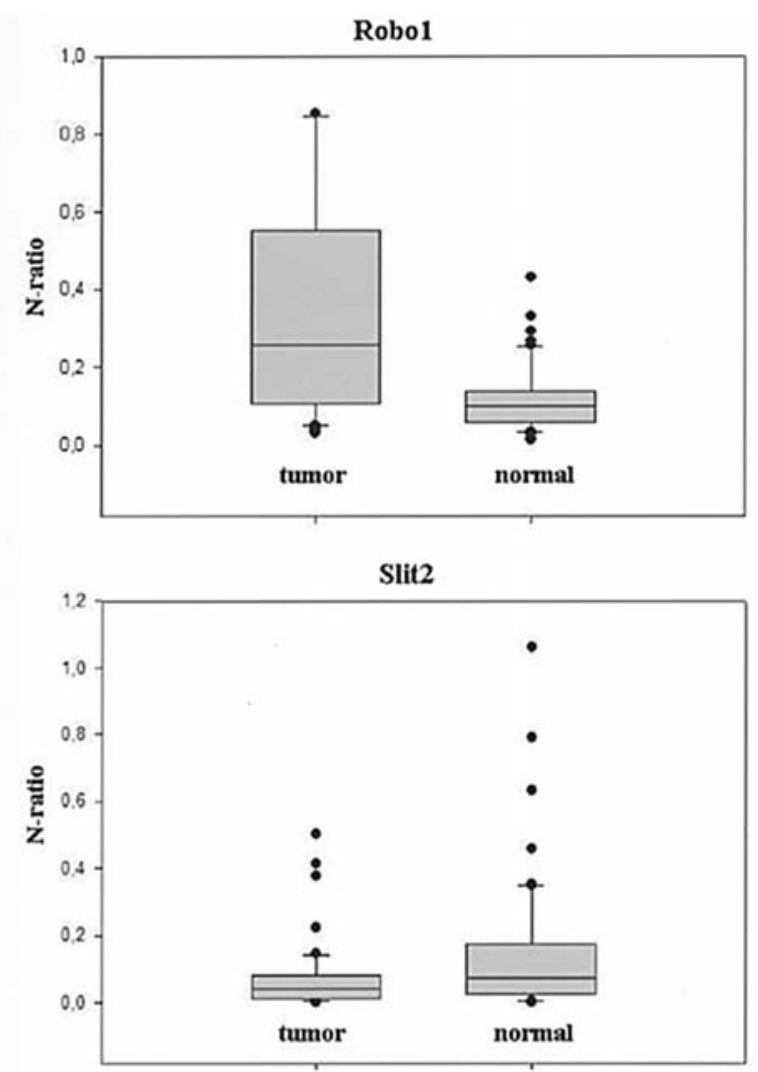

the collective was performed. In six cases, corresponding normal tissue was applicable. Immunohistochemical analyses (IHC) showed a cytoplasmatic staining of normal mucosa and

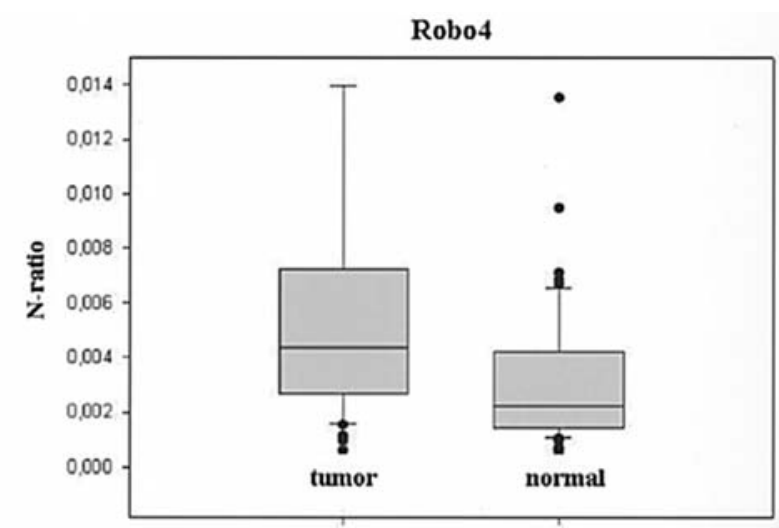

Figure 2. Results of expression analysis of Robo1, Robo4 and Slit2 in 50 CRC RNAs in duplicate by means of quantitative real-time-PCR analysis (LightCycler) confirm significant differential gene expression between normal and tumor samples for Robo1 $(\mathrm{p}<0.001)$ and also for Robo4 $(\mathrm{p}<0.01)$. Slit2 shows no significant differential expression $(\mathrm{p}>0.05)$. Box plots demonstrate median, upper and lower quartile, minimum and maximum values and outliers. With the use of relative quantification methods the results are expressed as a relative ratio (N-ratio) of the target of interest, to the housekeeping gene, 32 -microglobulin, measured in the same sample material.

tumor cells. In all ten samples, we saw a specific staining of tumor epithelial cells. Signal strength was estimated as strong $(+++)$ in four tumors, moderate $(++)$ in five, and weak $(+)$ in 
Table II. Evaluation of expression and localization of Robo1 in colorectal cancer by means of immunohistochemistry (IHC) and in situ hybridization. ${ }^{\mathrm{a}}$

\begin{tabular}{lcccccccc}
\hline $\begin{array}{l}\text { Patient no. } \\
(\mathrm{n}=10)\end{array}$ & UICC & Localization & $\begin{array}{c}\text { Stained tumor } \\
\text { cells }(\%)\end{array}$ & $\begin{array}{c}\text { Staining } \\
\text { intensity }\end{array}$ & $\begin{array}{c}\text { Stained normal } \\
\text { cells }\end{array}$ & $\begin{array}{c}\text { Staining } \\
\text { intensity }\end{array}$ & CD31 & In situ \\
\hline Co01 & II & Sigma & 90 & +++ & Positive & + & Positive & no \\
Co07 & II & Sigma & 80 & +++ & Positive & ++ & Positive & weak \\
Co15 & II & Sigma & 90 & +++ & NA & NA & Positive & weak \\
Co22 & III & Rectum & 100 & +++ & NA & NA & Positive & no \\
Co37 & II & Rectum & 100 & ++ & Positive & ++ & Positive & no \\
Co39 & II & Rectum & 90 & ++ & Positive & ++ & Positive & no \\
Co40 & II & Rectum & 100 & +++ & Positive & ++ & Positive & no \\
Co41 & II & Descendens & 100 & ++ & NA & NA & Positive & no \\
Co589 & I & Rectum & 70 & + & NA & NA & Positive & no \\
Co666 & III & Rectum & 100 & +++ & Positive & + & Positive & no \\
\hline
\end{tabular}

a Signal strength (staining intensity) was estimated as strong (+++), moderate (++), weak (+) and no staining (-). CD31 staining was performed as quality control for vascular endothelial cells. In four patients, no corresponding normal tissue was available (NA). In two patients, Robo1 in situ hybridization showed a cytoplasmatic expression and a weak staining of endothelial vessels in tumors compared to CD31 staining of vascular structures.

one tumor. A mean of $95 \%$ of tumor cells showed a specific staining and, in some cases, non-uniform distribution within the slides. We also observed specific staining of all normal mucosa samples. Evaluation of differential expression between normal and corresponding tumor tissues was analyzed in six out of ten patients. In two-thirds of corresponding samples (4/6), we detected a stronger staining in tumor vs. normal. In two matched pairs $(33.3 \%)$, normal and tumor tissues showed comparable staining intensity (Table II). In situ hybridization of Robo1 showed a cytoplasmatic expression and a weak staining of endothelial cells in two tumors and a comparatively stronger CD31 staining of the vascular vessels. ISH of Robo4 showed a specific staining of endothelial cells with moderate and strong signals solely along endothelial structures in tumor tissues in four tumor samples (Fig. 3). For Slit2, no specific binding, either in tumor tissue or in normal epithelial areas, was detected (results not shown).

\section{Discussion}

The guided migration of blood vessels and nerves often share common pathways suggesting that these processes follow similar molecular routes. Therefore, equivalent signal molecules are involved in sprouting angiogenesis and axonal guidance which has been demonstrated for netrins, semaphorins and ephrins (14-16). Slit/Robo signalling, initially identified in the axonal pathway finding, has been shown to play a role in the formation of blood vessels, particularly in vessels of tumors. This study provides insights into the function of Robo1, Robo4 and Slit2 in human colorectal cancer. We demonstrated the overexpression of the Robo genes in tumor tissue in comparison to adjacent normal tissue on the RNA level using Affymetrix microarray technology. These results were verified by subsequent real-time PCR analysis.
Only very limited information on the role of Robol in cancer is available. Studies on Robo1 in human prostate cancer exhibited a rather reduced expression compared to normal prostate tissue (4). Genetic knockout in mice led to inadequate lung development with bronchial hyperplasia implicating a role for Robo1 in lung cancer (17). Recently, Wang et al demonstrated a coherence of Robol and tumor angiogenesis in human melanoma. They detected Robo1 exclusively on human umbilical vein endothelial cells (HUVEC) and solely on vessels of malignant melanoma but not on vessels of normal tissue. Furthermore, microvessel density and tumor volume could be reduced by both a soluble form of Robol and a blocking antibody in a xenograft model (3).

Using cDNA microarrays, we were able to show that Robo1 is overexpressed in the tumor tissue of almost $50 \%$ of the patients included in this study. Those results were verified by real-time PCR analysis. Furthermore, by means of immunohistochemical staining with specific antibodies against Robo1, we localized the protein in tumor endothelial cells but most notably in tumor cells. It is interesting that Robo1 was overexpressed in CRC cells in contrast to the finding of Latil et al in prostate cancer. There seems to be a tissue specific expression pattern, and it can be hypothesized that Robol overexpression is produced by tumor and endothelial cells, and it may have an influence on the progression of CRC. Because of the constant up-regulation of Robol in tumors, this Robo receptor might be suitable as a target for anti-tumor therapy. Receptor inhibition using blocking antibodies or siRNA technology could be a reasonable therapeutic approach.

Robo4, also called magic roundabout, is a member of the Robo family, which has been determined because of its homology to Robo1 using in silico data mining (6). Despite the structural homology, there are particular differences regarding number and position of Ig and fibronectin domains. 

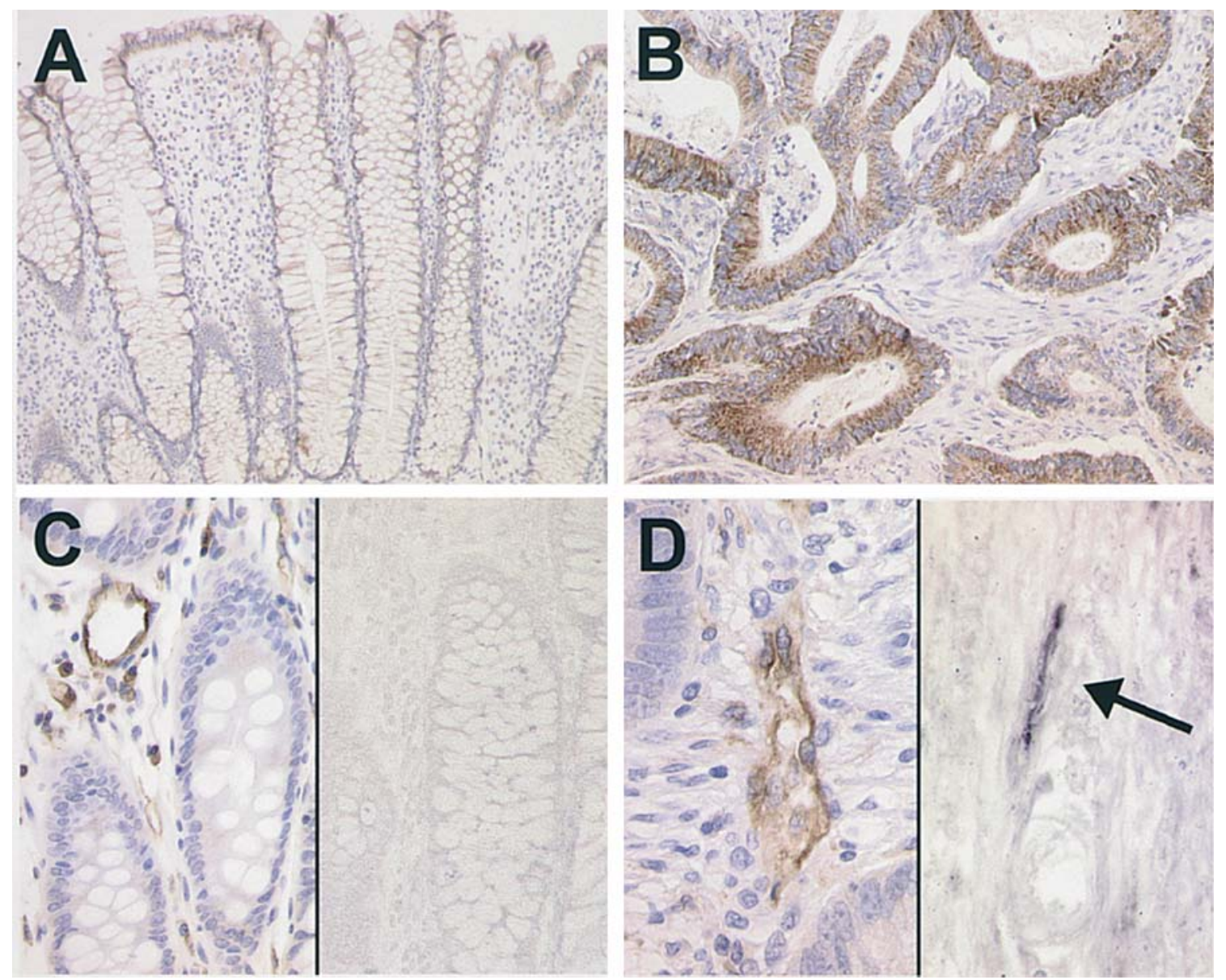

Figure 3. Immunohistochemical analysis showing weak to moderate cytoplasmic expression of Robo1 in (A) normal colonic epithelium compared to (B) colorectal carcinoma tumor cells which display strong cytoplasmic expression of Robo1. Negative controls were performed by omitting primary antibody. (C) CD31 expression of the endothelial cells in normal colonic mucosa (left panel) and negativity for Robo4 using in situ hybridization (right panel); (D) CD31 expression (left panel) and in situ hybridization showing focal Robo4 expression (arrow) in the endothelial cells of intratumoral vessels (right panel).

Accordingly, Robo receptors have been shown to be localized in different tissue compartments: Robo4 expression is limited to endothelial cells, whereas the other Robo receptors displayed activities in the nervous and immune system (18). The expression of Robo4 was located on vessels involved in the development of tumors, such as brain, breast, bladder, kidney and colon cancer (6). Moreover, recent studies reasoned that Robo4 seems to be essential in in vivo angiogenesis. Suchting et al demonstrated a blockade of angiogenic processes in a subcutaneous sponge model in vivo using a soluble extracellular domain of the receptor (19). Further investigation by Bedell et al demonstrated that knockdown of Robo4 leads to a loss of the coordinated vessel sprouting in a Zebrafish model (7).

In our studies, the expression analysis of Robo4 revealed an up-regulation in tumor tissue compared to normal epithelium. In comparison to Robo1 the dimension of up-regulation of Robo4 was much lower. Because of the lack of a commercially available antibody for Robo4, immunohistochemical staining was not feasible for localization of the protein in tissues. As the method of choice, in situ hybridization was performed, revealing an expression of Robo4 in the vessels of colorectal cancer tissue. In normal tissue, no binding of the specific probe was observed. Therefore, we assume that, as expected, Robo4 is primarily located on tumor vessels. The up-regulation of Robo4 in real-time expression analysis might result from an overexpression in endothelial cells also incorporated in the tumor tissue. Therefore Robo4 is a promising candidate for anti-angiogenic therapy.

In matters of Slit2, we demonstrated the expression of ligand in tumor and corresponding normal tissues by different techniques. While Affymetrix U133 results could not prove a significant deregulation between normal and tumor tissues, like for Robo1 and Robo4, possibly due to a higher ratio of absent calls, validation of array results by quantitative real-time-PCR revealed a trend towards down-regulation in tumor tissue. This can be interpreted as follows. Beside its accepted role in neurogenesis, Slit/Robo signalling has been detected in several tumor cell lines and in primary cancer (3-5). It has been demonstrated that Slit2 is frequently inactivated in breast and lung tumors by promoter region hypermethylation and allele loss (20). Furthermore, Dallol et al have shown that Slit2 is also inactivated in colorectal cancer and suppresses growth of colorectal carcinoma cells. They suggest that the presence of Slit2 reduces tumor growth by actively inducing apoptosis and they have nominated Slit2 for tumor suppressor gene for sporadic colorectal cancer $(8,21,22)$. Inactivation or, as we demonstrated, reduced relative expression of a potential tumor suppressor 
gene in colorectal cancer tissue may support tumor growth and explain the role of Slit2 in cancer development. In connection with our synchronous observation of up-regulation of the Slitreceptors, Robo1 and Robo4, in tumor tissue, a lower gene expression of the ligand, Slit2, possibly affects a reactive upregulation of Slit-receptors. Wang et al (3) identified Slit2 as a chemoattractive molecule for Robo1 localized on endothelial cells that is secreted by tumor cells. On the contrary, we did not detect any specific binding of the Slit2-antibody either in tumor tissue or in normal epithelial areas. This observation corresponds to our findings on the RNA-level. Therefore, we could not support the hypothesis of Slit2 as an attractant in tumor angiogenesis of colorectal cancer. The role of Slit2 in colorectal cancer remains an open book. It is not clear if Slit2 is up- (attractant) or down-regulated (tumor suppressor) in CRC. Finally, due to the lower expression in colorectal cancer demonstrated by our group, it is possible that Slit2 is irrelevant and that there may be ligands other than Slit2 which are responsible for tumorgenesis in colorectal cancer.

The present study is the first to report the analysis of Robo1, Robo4 and Slit2 in human colorectal cancer. In our study, we demonstrated an up-regulation of Robo1 and Robo4 in colorectal cancer tissue. Furthermore, histological analysis demonstrated the expression of both receptors in tumor epithelium and vascular vessels, whereas protein expression of Robo4 was restricted to endothelial structures in tumor tissue. Therefore, the Robo proteins provide potential target structures for the anti-tumorigenic and anti-angiogenic therapy of colorectal carcinoma.

\section{Acknowledgements}

Microarray studies have been performed in cooperation between the Department of Surgery and metaGen Pharmaceuticals GmbH i.L. (Berlin, Germany).

\section{References}

1. Vargesson N, Luria V, Messina I, Erskine L and Laufer E: Expression patterns of Slit and Robo family members during vertebrate limb development. Mech Dev 106: 175-180, 2001

2. Wong K, Park HT, Wu JY and Rao Y: Slit proteins: molecular guidance cues for cells ranging from neurons to leukocytes. Curr Opin Genet Dev 12: 583-591, 2002.

3. Wang B, Xiao Y, Ding BB, et al: Induction of tumor angiogenesis by Slit-Robo signaling and inhibition of cancer growth by blocking Robo activity. Cancer Cell 4: 19-29, 2003.

4. Latil A, Chene L, Cochant-Priollet B, Mangin P, Fournier G, Berthon $\mathrm{P}$ and Cussenot O: Quantification of expression of netrins, slits and their receptors in human prostate tumors. Int J Cancer 103: 306-315, 2003.
5. Prasad A, Fernandis AZ, Rao Y and Ganju RK: Slit proteinmediated inhibition of CXCR4-induced chemotactic and chemoinvasive signaling pathways in breast cancer cells. J Biol Chem 279: 9115-9124, 2004.

6. Huminiecki L, Gorn M, Suchting S, Poulsom R and Bicknell R: Magic roundabout is a new member of the roundabout receptor family that is endothelial specific and expressed at sites of active angiogenesis. Genomics 79: 547-552, 2002.

7. Bedell VM, Yeo SY, Park KW, et al: Roundabout4 is essential for angiogenesis in vivo. Proc Natl Acad Sci USA 102: 6373-6378, 2005.

8. Dallol A, Morton D, Maher ER and Latif F: SLIT2 axon guidance molecule is frequently inactivated in colorectal cancer and suppresses growth of colorectal carcinoma cells. Cancer Res 63: 1054-1058, 2003.

9. Fernando NH and Hurwitz HI: Targeted therapy of colorectal cancer: clinical experience with bevacizumab. Oncologist 9 (suppl 1): 11-18, 2004.

10. Kasper G, Vogel A, Klaman I, et al: The human LAPTM4b transcript is upregulated in various types of solid tumours and seems to play a dual functional role during tumour progression. Cancer Lett 224: 93-103, 2005.

11. Rubie C, Kempf K, Hans J, et al: Housekeeping gene variability in normal and cancerous colorectal, pancreatic, esophageal, gastric and hepatic tissues. Mol Cell Probes 19: 101-109, 2005.

12. Bhargava S, Stummeyer T, Hotz B, Hines OJ, Reber HA, Buhr HJ and Hotz HG: Selective inhibition of endothelin receptor A as an anti-angiogenic and anti-proliferative strategy for human pancreatic cancer. J Gastrointest Surg 9: 703-709, 2005.

13. Varis A, Zaika A, Puolakkainen P, et al: Coamplified and overexpressed genes at ERBB2 locus in gastric cancer. Int $\mathbf{J}$ Cancer 109: 548-553, 2004.

14. Klein R: Excitatory Eph receptors and adhesive ephrin ligands. Curr Opin Cell Biol 13: 196-203, 2001.

15. Torres-Vazquez J, Gitler AD, Fraser SD, et al: Semaphorinplexin signaling guides patterning of the developing vasculature. Dev Cell 7: 117-123, 2004.

16. Park KW, Crouse D, Lee M, et al: The axonal attractant Netrin-1 is an angiogenic factor. Proc Natl Acad Sci USA 101: 16210-16215, 2004

17. Xian J, Clark KJ, Fordham R, Pannell R, Rabbitts TH and Rabbitts PH: Inadequate lung development and bronchial hyperplasia in mice with a targeted deletion in the Dutt1/Robo1 gene. Proc Natl Acad Sci USA 98: 15062-15066, 2001.

18. Mailhos C, Modlich U, Lewis J, Harris A, Bicknell R and Ish-Horowicz D: Delta4, an endothelial specific notch ligand expressed at sites of physiological and tumor angiogenesis. Differentiation 69: 135-144, 2001.

19. Suchting S, Heal P, Tahtis K, Stewart LM and Bicknell R: Soluble Robo4 receptor inhibits in vivo angiogenesis and endothelial cell migration. FASEB J 19: 121-123, 2005.

20. Dallol A, Da Silva NF, Viacava P, Minna JD, Bieche I, Maher ER and Latif F: SLIT2, a human homologue of the Drosophila Slit2 gene, has tumor suppressor activity and is frequently inactivated in lung and breast cancers. Cancer Res 62: 5874-5880, 2002.

21. Stein E and Tessier-Lavigne M: Hierarchical organization of guidance receptors: silencing of netrin attraction by slit through a Robo/DCC receptor complex. Science 291: 1928-1938, 2001.

22. Forcet C, Ye X, Granger L, Corset V, Shin H, Bredesen DE and Mehlen P: The dependence receptor DCC (deleted in colorectal cancer) defines an alternative mechanism for caspase activation. Proc Natl Acad Sci USA 98: 3416-3421, 2001. 